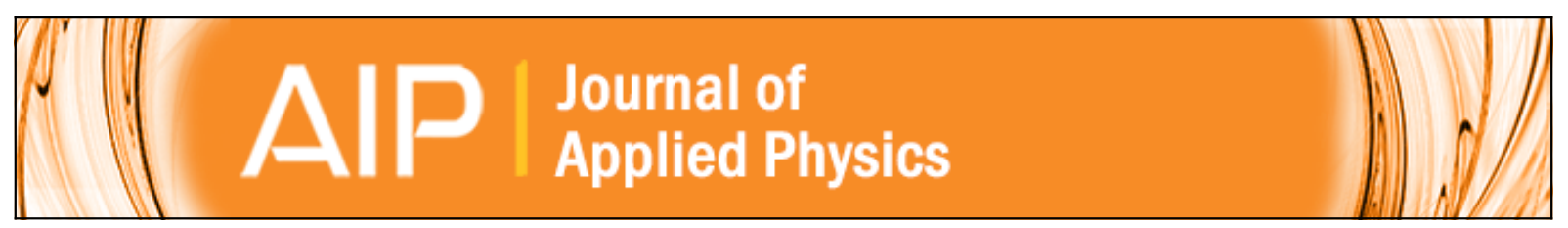

Imaging the ultrafast Kerr effect, free carrier generation, relaxation and ablation dynamics of Lithium Niobate irradiated with femtosecond laser pulses

Mario Garcia-Lechuga, Jan Siegel, Javier Hernandez-Rueda, and Javier Solis

Citation: Journal of Applied Physics 116, 113502 (2014); doi: 10.1063/1.4895833

View online: http://dx.doi.org/10.1063/1.4895833

View Table of Contents: http://scitation.aip.org/content/aip/journal/jap/116/11?ver=pdfcov

Published by the AIP Publishing

AIP $\mid$ Applied Physics Letters

is pleased to announce Reuben Collins as its new Editor-in-Chief

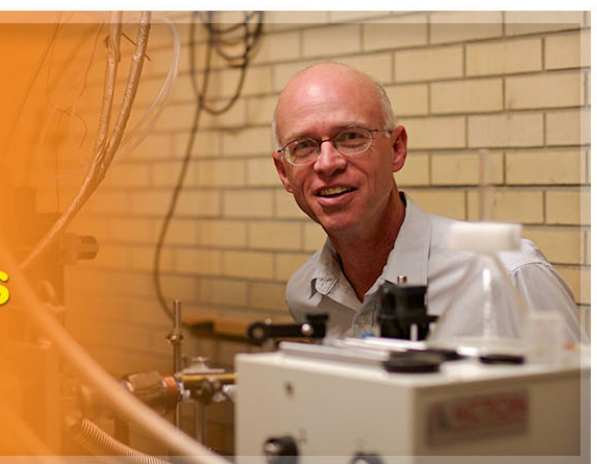




\title{
Imaging the ultrafast Kerr effect, free carrier generation, relaxation and ablation dynamics of Lithium Niobate irradiated with femtosecond laser pulses
}

\author{
Mario Garcia-Lechuga, ${ }^{\text {a) }}$ Jan Siegel, ${ }^{\text {b) }}$ Javier Hernandez-Rueda, and Javier Solis \\ Laser Processing Group, Instituto de Optica, CSIC, Serrano 121, 28006 Madrid, Spain
}

(Received 8 August 2014; accepted 4 September 2014; published online 16 September 2014)

\begin{abstract}
The interaction of high-power single 130 femtosecond (fs) laser pulses with the surface of Lithium Niobate is experimentally investigated in this work. The use of fs-resolution time-resolved microscopy allows us to separately observe the instantaneous optical Kerr effect induced by the pulse and the generation of a free electron plasma. The maximum electron density is reached $550 \mathrm{fs}$ after the peak of the Kerr effect, confirming the presence of a delayed carrier generation mechanism. We have also observed the appearance of transient Newton rings during the ablation process, related to optical interference of the probe beam reflected at the front and back surface of the ablating layer. Finally, we have analyzed the dynamics of the photorefractive effect on a much longer time scale by measuring the evolution of the transmittance of the irradiated area for different fluences below the ablation threshold. (C) 2014 AIP Publishing LLC. [http://dx.doi.org/10.1063/1.4895833]
\end{abstract}

\section{INTRODUCTION}

Lithium Niobate $\left(\mathrm{LiNbO}_{3}\right)$ is an interesting dielectric material with exceptional electrical and optical properties of use in a large number of applications in integrated optics and photonics. ${ }^{1}$ In order to study its response upon irradiation with ultrashort and intense laser pulses, several groups performed time-resolved experiments using different pump and probe schemes. Transmission experiments ${ }^{2-5}$ were focused on the study of laser-induced electron plasma relaxation and the long-lasting optical response associated to the photorefractive effect. Diffraction techniques ${ }^{6-8}$ were applied to study optical effects associated to Kerr non-linearities and the generation of free electron plasma.

These works were limited to fluences below the ablation threshold. In other dielectrics, ${ }^{9}$ the comprehension of the excitation, relaxation and structural transformation dynamics has been analyzed by experiments involving surface ablation. Over the last fifteen years, experimental and theoretical works have been devoted to this purpose that still has a number of controversial aspects. Among them, the relative roles of multiphoton, impact and avalanche ionization in the generation of free carriers is still under debate and an active field of research. ${ }^{10-15}$ With these motivations, we have analyzed the interaction of $\mathrm{LiNbO}_{3}$ with fs-laser pulses with peak fluences above the ablation threshold. For this purpose, we have used an imaging (microscopy), pump-and-probe technique. This technique provides images of the material surface at different time delays after being irradiated; thus combining ultrafast time resolution with microscopic spatial (and thus local fluence) resolution.

In the present work, we observe and analyze the instantaneous non-linear Kerr effect in $\mathrm{LiNbO}_{3}$ and exploit it for a

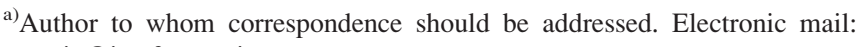
mario@io.cfmac.csic.es

b)j.siegel@io.cfmac.csic.es.
}

precise determination of the zero delay between the pump and probe pulses. This precise timing is necessary for a correct interpretation of the electron excitation and relaxation dynamics. Also, we observe over a time lapse from a few tens to several hundreds of picoseconds after excitation a characteristic ring pattern that dynamically changes for increasing delay times. This exotic optical effect during the ablation process was first observed in metals and semiconductors by Sokolowski-Tinten and coworkers ${ }^{16,17}$ and explained as the consequence of interference of the probe beam reflected at the two interfaces of the ablating layer. In recent works, ${ }^{18,19}$ we have observed this effect for the first time in several dielectric materials, which indicates that ablation may have a common thermodynamic path for all solid materials. Finally, we analyze transient absorption effects in $\mathrm{LiNbO}_{3}$ in the subablative fluence regime that are related to the photorefractive effect.

\section{EXPERIMENTAL}

The sample used in this study is a commercial, $10 \mathrm{~mm}$ thick, single crystal of undoped $\mathrm{LiNbO}_{3}$ supplied by VMTIM Germany, with an optical bandgap of $\sim 3.5 \mathrm{eV}$. The laser used for irradiation is a Ti:sapphire femtosecond amplifier (Spitfire pro, Spectra Physics), which produces transform-limited pulses of $130 \mathrm{fs}$ FWHM (Full Width at Half Maximum) at $800 \mathrm{~nm}$. The temporal pulse shape was characterized with a home-made ${ }^{20}$ frequency-resolved optical gating system in polarization gate (PG-FROG) configuration, as described by Trebino et al. ${ }^{21}$

Irradiations were performed with an s-polarized beam using single pulses with a constant peak fluence $\left(2.12 \mathrm{~J} / \mathrm{cm}^{2}\right)$ after being focused at the $\mathrm{XY}$ surface of $\mathrm{LiNbO}_{3}$, where the $\mathrm{c}$-axis is parallel to the $\mathrm{Z}$ direction. Pump pulses reached the surface at an angle of $53^{\circ}$, producing an elliptical excitation spot with a measured Gaussian intensity distribution $\left(59 \mu \mathrm{m} \times 96.8 \mu \mathrm{m} 1 / \mathrm{e}^{2}\right.$-diameters $)$. Under these experimental 
conditions we determined, using the Liu method, ${ }^{22}$ an ablation threshold fluence $F_{t h}=1.55 \mathrm{~J} / \mathrm{cm}^{2}$.

In order to study the temporal evolution of the laserinduced processes, we used a femtosecond microscopy set up, as described in detail in a previous work ${ }^{23}$ and sketched in Figure 1. Briefly, a fraction of the pump pulse was frequency-doubled ( $400 \mathrm{~nm}$, probe pulse) by a barium borate crystal (BBO) and used as illumination source, performing in-situ microscopy of the irradiated region with a long distance objective lens (NA 0.42). This pump-and-probe technique allowed us to record images of the surface at different delays after the laser excitation with sub-picosecond and micrometer resolution. Two different microscopy configurations were used in this work, reflection and transillumination. In reflection, a prism compressor ${ }^{24}$ was used in order to pre-compensate the group velocity dispersion (GVD) experienced by the probe pulse in its pass through the optical elements of the set-up. After adjusting the distance of the two prisms in the compressor probe pulses of $\sim 150$ fs duration were achieved. In trans-illumination, the highly dispersive thick sample impedes performing a sufficient GVD pre-compensation of the probe pulse and the thus the compressor was not used and the temporal resolution limited to $1 \mathrm{ps}$.

The delay between pump and probe pulses was controlled by a motorized optical delay line, with a minimum step size set to $50 \mathrm{fs}$. We took as the experimental zero delay value the position of the optical delay line corresponding to $50 \mathrm{fs}$ before the first observable change in the surface reflectivity occurs. The maximum delay achieved with the motorized delay line is $1 \mathrm{~ns}$. For longer delays additional mirrors were used to generate discrete delay steps.

\section{RESULTS AND DISCUSSION}

This section is divided in three parts corresponding to the different characteristic time scales analyzed, (a) ultrafast excitation processes at the early stage of the interaction $(<1 \mathrm{ps})$,

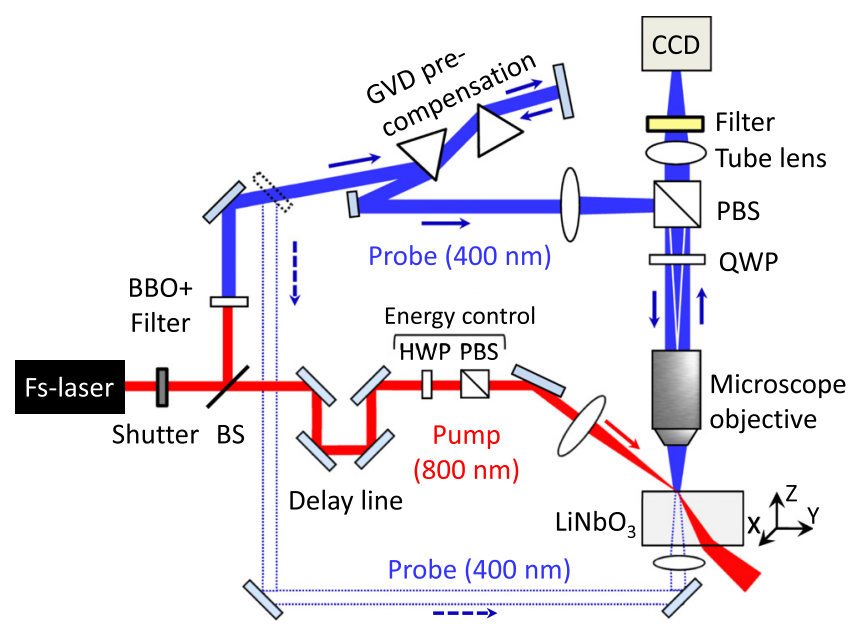

FIG. 1. Sketch of the irradiation and the two configurations of the femtosecond microscopy set-up, in reflection and in trans-illumination (dotted lines), where the PBS and QWP before the CCD-camera are removed. BS: Beam Splitter; PBS: Polarizing Beam-Splitter Cube; HWP: Half-Wave Plate; QWP: Quarter-Wave Plate; CCD: charge-coupled device (Camera). (b) interaction of the probe beam with the ablating material and appearance of transient Newton rings (from tens of ps to $\mathrm{ns}$ ), (c) slow relaxation processes lasting up to the millisecond time scale.

\section{A. Nonlinear Kerr effect and free carrier generation}

Time-resolved microscopy experiments allow us to visualize how the reflectivity of the surface evolves on ultrashort time-scales. Figure 2 shows several images of the surface for delays between the pump and probe pulses ranging from $50 \mathrm{fs}$ to $600 \mathrm{fs}$. The images shown are images obtained after dividing the corresponding single image recorded at a given delay by a single image recorded, at the same sample position, before exposure to the pump laser pulse. Using this method, spatial inhomogeneities in the illumination field are efficiently reduced and the initial reflectivity and transmission values for each pixel are normalized to 1 .

We can see that at the center of the irradiated region, where the local fluence is maximum, the reflectivity initially decreases before undergoing a subsequent increase, reaching a high value for a delay of $600 \mathrm{fs}$, which is related to free electron generation. Interestingly, the reflectivity behavior observed in the outer region of the irradiated zone is the opposite, with an initial fast increase followed by a subsequent decrease. A comparison of these images with the crater profile also shown in Figure 2, reveals that most of the high reflectivity ring observed during the first $\sim 300$ fs delay lies outside the ablated crater. These annular ultrafast transient reflectivity changes occur in a subablative regime, which suggests that we are imaging the non-linear optical Kerr response of the material. A previous work on the interaction
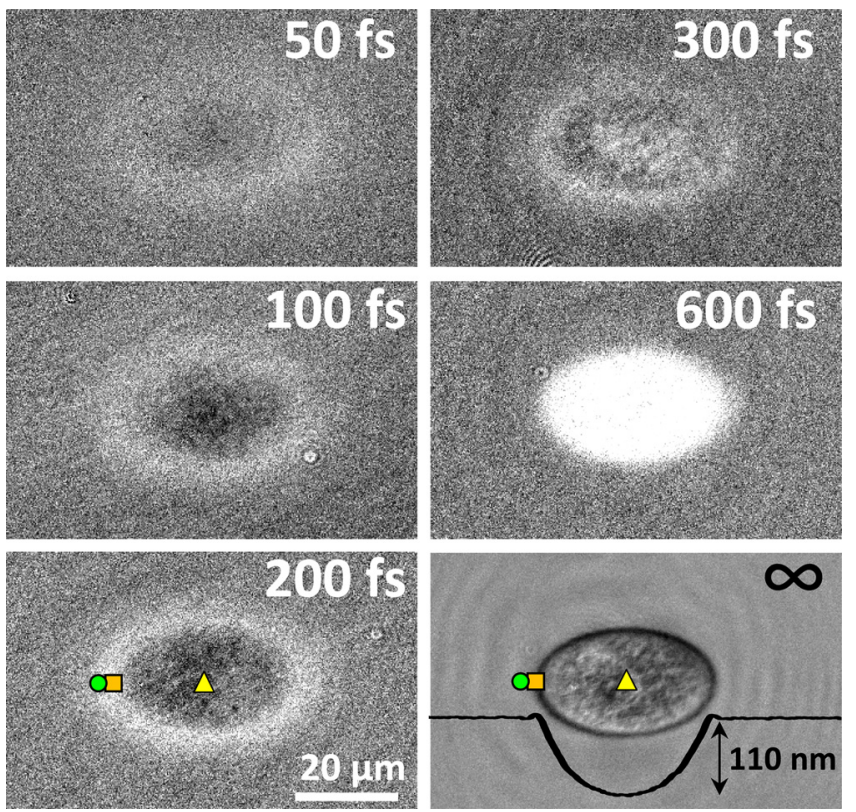

FIG. 2. Time-resolved surface reflectivity images $\left(\lambda_{\text {probe }}=400 \mathrm{~nm}\right)$ of Lithium Niobate at short delay times (see labels) after exposure to a pump pulse $\left(\lambda_{\text {pump }}=800 \mathrm{~nm}, 130 \mathrm{fs}, 2.12 \mathrm{~J} / \mathrm{cm}^{2}\right)$ incident at $53^{\circ}$. The figure labeled as infinite (some seconds after irradiation) includes as an inset a transverse cross section of the ablated crater depth, measured with an interferometric microscope. Yellow triangles, orange squares and green circles correspond to local fluences equal to $2.12,1.50$ and $1.40 \mathrm{~J} / \mathrm{cm}^{2}$, respectively. 
between fs-laser pulses and $\mathrm{LiNbO}_{3}$ (Ref. 6) demonstrated the coexistence during the interaction of non-linear optical Kerr effect and free carriers generation, yet without spatially resolving them. With the aim of studying the temporal and fluence dependence of each effect, in Figure 3(a) a comparison of the temporal evolution of the reflectivity for different local fluences is shown. The experimental points were obtained by radial averaging analysis of the images, taking into account the elliptic shape of the excited region. ${ }^{25}$

In Figure 3(a), we show the reflectivity evolution for two different local fluences below the ablation threshold where the Kerr effect should be predominant. For the local fluence closer to the ablation threshold, $1.50 \mathrm{~J} / \mathrm{cm}^{2}$, we observe that the reflectivity after the maximum does not return to the level before irradiation within the temporal window shown. This suggests that for very short delays the non-linear Kerr effect dominates the material response but competes with the effects associated to the generation of free electrons. For the lower fluence, $1.40 \mathrm{~J} / \mathrm{cm}^{2}$, the reflectivity almost returns to the initial value (non-irradiated surface) indicating that during the pump pulse duration free carrier effects are negligible with respect to the Kerr effect. The

(a)

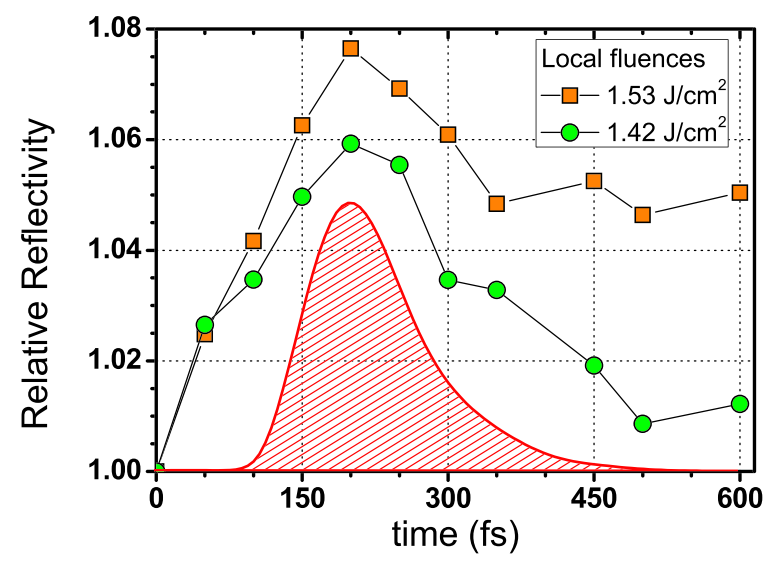

(b)

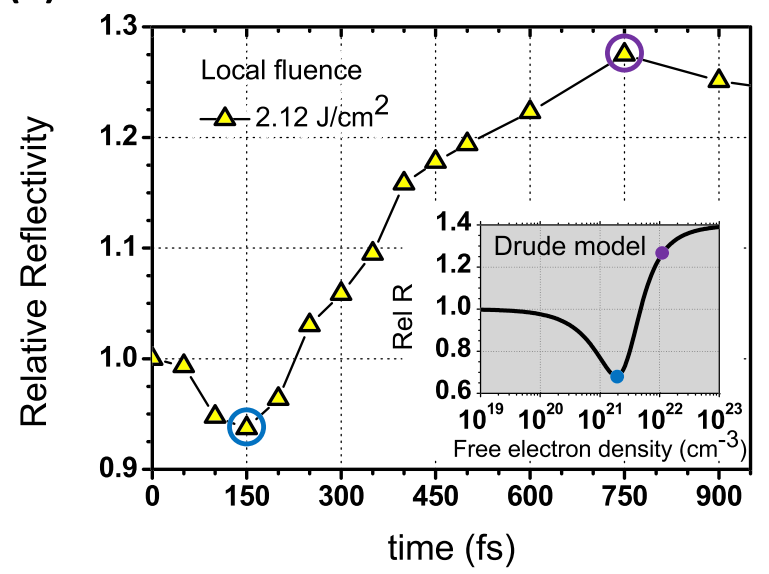

FIG. 3. Transient reflectivity curves extracted from time-resolved images. (a) At two local fluences slightly below the ablation threshold. The inset is the temporal profile of the pump pulse obtained by PG-FROG. (b) Above the ablation threshold at the center of the irradiation spot. The inset shows a simulation of the surface reflectivity as a function of the free electron density using the Drude model. reflectivity change at the surface caused by the pump-pulse induced non-linear Kerr effect and measured by the probe pulse can, in fact, be considered a cross-correlation measurement, in which the probe pulse is slightly broader (by dispersion) than the pump pulse. By definition, is the mathematical convolution of both pulse forms and therefore broader than the individual pulses. This is the reason why the optical signal measured is slightly broader than the pump pulse shape retrieved from a PG-FROG measurement, also plotted in Figure 3(a).

The maximum reflectivity increase due to the Kerr effect, $\Delta R=5.9 \%$, is reached for a local irradiance, $I=10.1$ $\mathrm{TW} / \mathrm{cm}^{2}$ (local fluence of $1.40 \mathrm{~J} / \mathrm{cm}^{2}$, Gaussian pulse of 130fs FWHM). By using the relationship between the reflectivity and the refractive index,

$$
R=\left|\frac{n-1}{n+1}\right|^{2}
$$

a refractive index increase of $\Delta n=0.074$ is obtained, with $R_{0}=0.175$ and $n_{0}=2.44$ (at $\lambda=400 \mathrm{~nm}$ ). ${ }^{26}$ Following the Kerr effect dependence of the refractive index with the irradiance:

$$
n=n_{0}+n_{2} I,
$$

the non-linear refractive index estimated is $n_{2}(\lambda=400 \mathrm{~nm})$ $\approx 7 \cdot 10^{-6} \mathrm{~cm}^{2} / \mathrm{GW}$. This value is consistent with previously reported values obtained using non-imaging techniques: $n_{2}(\lambda=532 \mathrm{~nm})=5.3 \cdot 10^{-6} \mathrm{~cm}^{2} / \mathrm{GW}^{27}$ (z-scan) and $n_{2}(\lambda$ $=776 \mathrm{~nm}) \gg 10 \cdot 10^{-6} \mathrm{~cm}^{2} / \mathrm{GW}^{7}$ (transient diffraction technique).

It is worth noting that the non-linear Kerr effect occurs only while the intense electric field is present. For the pulse duration used, over the temporal spread of the pulse and some hundreds of fs after, the photo-generated carriers are still decoupled from the lattice as the phonon emission time is considerably longer. As a consequence thermal effects can be disregarded in the ultrafast optical response of the material over the sub-picosecond scale.

Figure 3(b) shows the reflectivity evolution at the spot center, where the local fluence $\left(F=2.12 \mathrm{~J} / \mathrm{cm}^{2}\right)$ is above the ablation threshold and thus corresponds to the regime in which generation of free electrons determines the optical response. We have performed a simulation based on the Drude model of the evolution of the reflectivity at $\lambda=400 \mathrm{~nm}$ when the electron density increases, using a damping constant inversely proportional to the photogenerated carrier density, as detailed in Refs. 13 and 28, with $\tau_{l}=1$ fs (inset of Figure $3(b))$. The shape of both (experimental and modeled) curves is essentially similar, featuring an initial drop in reflectivity followed by a steep rise caused by the increase of free carrier density. However, the initial reflectivity drop is less pronounced in the experimental data. This is caused by two reasons. The first one is the limited time resolution of the experiment. The second and most important one is that the Kerr effect is also present in the central region of the irradiated spot, producing an increase of the reflectivity. Kerr effect and free carrier generation are independent phenomena 
contributing to the reflectivity change in opposite directions. When each effect will be dominant depends on local fluence and time of observation.

An important observation derived from these results is that the reflectivity peak is reached $550 \mathrm{fs}$ after the pump pulse intensity peak (maximum of Kerr effect). This observation implies that free electron generation occurs also after the end of the excitation pulse, via a delayed electron excitation mechanism, such as impact ionization. This issue has led in the past to a vivid debate and controversial results. ${ }^{10,12,13,29}$ Our study, with a precise and reliable determination of the zero delay based on the observation of the Kerr effect, and direct space-and-fluence resolved measurements, confirms the presence of delayed carrier generation, at least in $\mathrm{LiNbO}_{3}$.

\section{B. Transient interference effects at the ablating layer}

In Figures 4(a) and 4(b), we show several micrographs recorded at some hundreds of picoseconds time delay in reflection and in trans-illumination, featuring a characteristic ring structure inside the ablating region. The process, as depicted in Figure 4(c), is related to optical interference between the probe beam reflected at the front and back surfaces of the ablating layer, forming these transient Newton rings. ${ }^{18,19}$

The expansion of the ablating layer is accompanied by an increase of the number of rings which can be observed in both imaging modes. The period for a given delay is the same in reflection and in transmission, but maxima and minima are interchanged (Figure 4(d)), confirming the interferential origin of the phenomenon. For both imaging configurations, the same fluence was used and the final craters are identical. Transmittance images might look smaller, because the pattern begins with a thin bright ring (constructive interference) instead of a dark ring in reflection illumination (destructive interference) (see Figure 4(d)). Another

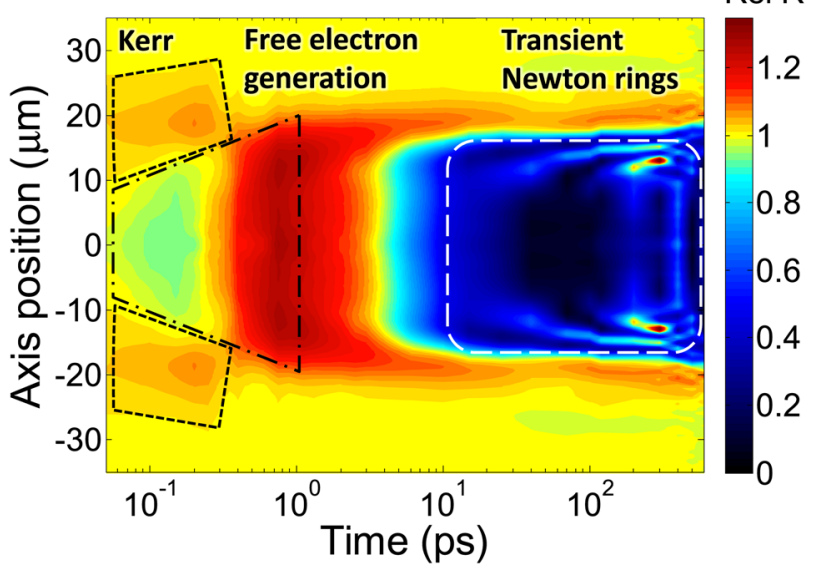

FIG. 5. Representation of the spatial distribution of the reflectivity as a function the of delay time. The color scale indicates the reflectivity value at each position and delay. The areas enclosed in the different regions correspond to reflectivity changes associated with different predominant effects in different temporal/fluence scales. The spatial positions can be directly correlated to local fluence values: $0 \mu \mathrm{m}, 10 \mu \mathrm{m}, 20 \mu \mathrm{m}$, and $30 \mu \mathrm{m}$-distance from spot center correspond to fluences of $2.12,1.95,1.51$, and $0.98 \mathrm{~J} / \mathrm{cm} 2$, respectively.

difference is the amplitude of maxima and minima. In reflectivity the resulting optical contrast is higher than in transmission. This is caused by the fact that in transmission the intensity of the two interfering beams is very different.

All effects observed during interaction are summarized in a transient reflectivity-local fluence map ${ }^{25}$ shown in Figure 5. This representation of the temporal evolution of the surface reflectivity helps in visualizing the characteristic time scales and local fluence regimes (related to spatial position) of the different processes involved: non-linear Kerr effect, free electron plasma formation, electron relaxation (energy transference to the lattice via electron-phonon coupling and other relaxation mechanisms, like trapping), and material ablation.

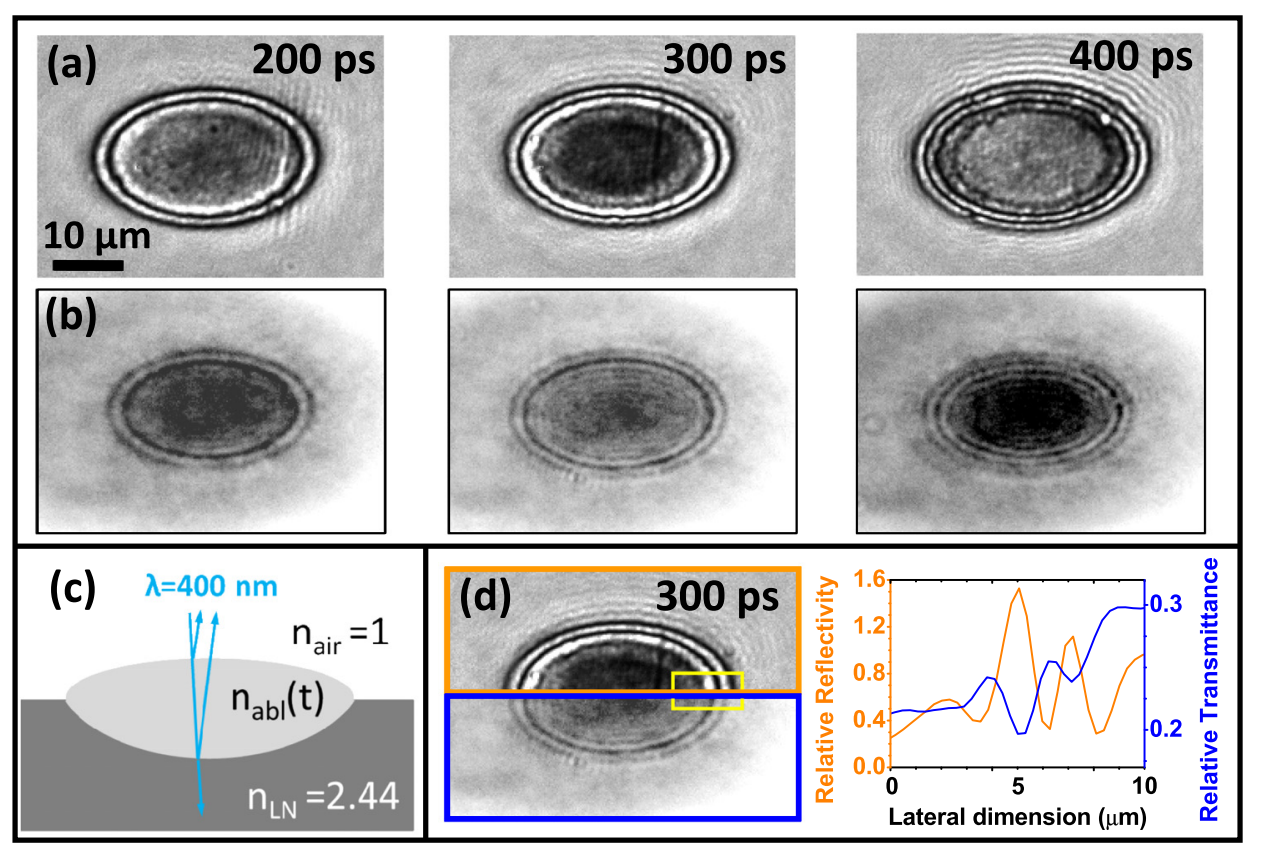

FIG. 4. Images of the irradiated region at different delays (see labels) showing the presence of transient Newton rings in reflection, row (a), and in transillumination, row (b). The contrast has been enhanced to better visualize the ring structure. (c) Scheme of the formation of the transient Newton rings. (d) Comparison between images in reflection and transmission at a delay of $300 \mathrm{ps}$, and reflectivity profiles of the square marked in (d), where transient Newton rings are observed. 


\section{Photorefractive effect below the ablation threshold}

In the microscopy images in trans-illumination mode (c.f. Figure 4(b)), a relatively large dark region around the ablated region remains long after the irradiation for local fluences well below the ablation threshold. This sub-ablative effect is due to one of the most relevant properties of $\mathrm{LiNbO}_{3}$, photorefractivity, which can be triggered by $\mathrm{cw}$ light as well as ultrashort laser pulses. ${ }^{2}$ The origin of this effect in ultrashort studies, thoroughly described in the literature, ${ }^{4,30}$ is that photo-generated carriers can be temporally trapped in intermediate levels in the bandgap, caused by antisites that originate from partial disorder of the crystal structure or points defects. The lifetime of this intermediate levels depends on the light intensity, the light wavelength, and the crystal temperature. ${ }^{31}$ In the transmission images (Figure 6(a)), photorefractivity is evidenced by a change in the transmittance, where the long lasting effect can be still appreciated in the image recorded at 43 ns delay. In order to study the temporal evolution and the fluence dependence of photorefractivity, a radial analysis of the transmittance images was performed. As the pump pulses reach the surface at an angle of $53^{\circ}$ only the right side of the images was analyzed. The left side of the image contains contributions from the modifications produced inside the material caused by the propagating beam, which appear defocused and on the left (c.f. Figure 1). Figure 6(b) shows the time evolution of the transmittance at different local fluences. The last data points, at

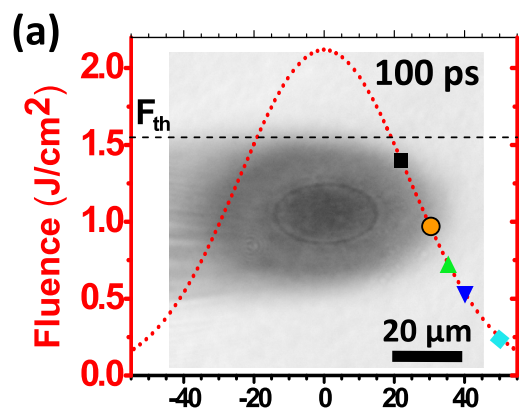

43 ns

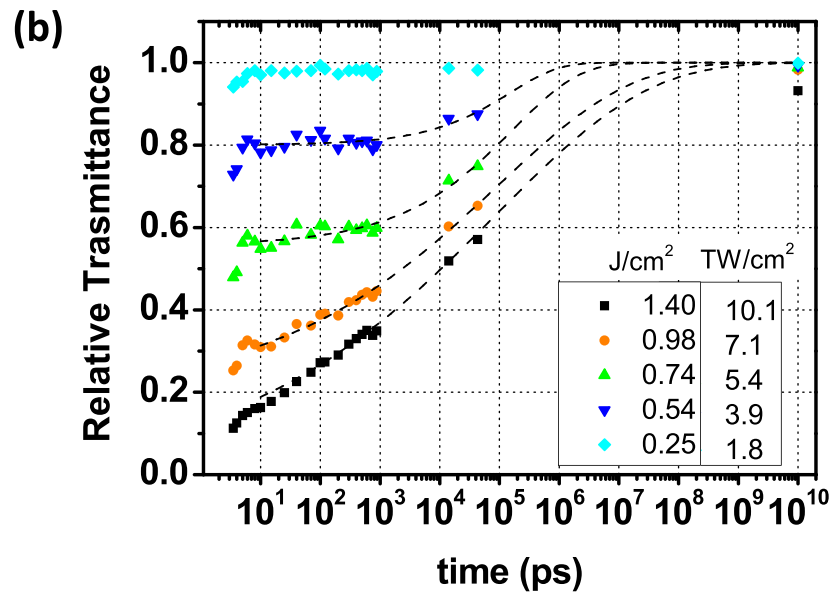

FIG. 6. (a) Time-resolved transmission images and Gaussian spatial intensity distribution of the pump beam (the contrast has been enhanced to better visualize the dark region). Symbols indicate the sub-ablative local fluences of the curves in (b), showing the evolution of the transmittance versus time. Dashed lines correspond to the fit of the experimental data to a stretchedexponential function.
$10 \mathrm{~ms}$ delay time, were obtained by irradiating with a pump pulse and illuminating the excited region with the following pulse exiting the amplifier $(100 \mathrm{~Hz}$ repetition rate).

In the $3.5-10 \mathrm{ps}$ interval, the transmittance curves are slightly different for the various local fluences when compared to the behavior at longer delays. This is caused by the free electrons remaining in the conduction band that finally thermalize or are trapped. From 10 ps onwards the experimental data have been fitted to a stretched-exponential function, ${ }^{31}$

$$
T(t)=1-T(t=0) \cdot \exp \left[-(t / \tau)^{\beta}\right]
$$

where $\beta$ is an exponent between 0 and 1 and $\tau$ is the characteristic decay time. For local fluences well below the ablation threshold $\left(0.74 \mathrm{~J} / \mathrm{cm}^{2}\right.$ and $\left.0.54 \mathrm{~J} / \mathrm{cm}^{2}\right)$ we obtained $\tau=150 \pm 30 \mathrm{~ns}$ and $\beta=0.40 \pm 0.15$, where the considerable error is due to the small transmission changes observed before $1 \mathrm{~ns}$ and the few experimental points available afterwards. The optical response is similar to the one reported by Ashihara et al..$^{32}$ using a non-imaging technique for studying the absorption of $\mathrm{LiNbO}_{3}$ upon irradiation with a polarized beam perpendicular to c-axis, where the fast component parameters of the fit to a doubled stretched-exponential were $\tau=150 \mathrm{~ns}$ and $\beta=0.27$.

For fluences close to the ablation threshold $\left(1.40 \mathrm{~J} / \mathrm{cm}^{2}\right.$ and $0.98 \mathrm{~J} / \mathrm{cm}^{2}$ ), we can see that the irradiated region becomes almost opaque and that the relaxation time is faster than for lower fluences, leading to values of $\tau=$ $90 \pm 5 \mathrm{~ns}$ and $\beta=0.18 \pm 0.04$. The only work with similar experimental parameters (pump at $800 \mathrm{~nm}$, probe at $400 \mathrm{~nm}$ and fluences close to the ablation threshold but inside the material) we are aware of is the one by Gamaly et al. ${ }^{2}$ However, they only report an observation by an ordinary video camera of the relaxation time and estimate it to be a few hundreds of milliseconds without a clear assignment of the local fluence.

\section{CONCLUSIONS}

We have investigated the interaction of single fs-pulses with single crystal Lithium Niobate using a time-resolved imaging technique. We have temporally and fluenceresolved the optical changes produced by the ultrafast Kerr effect, photo-excited carriers and the long-lasting photorefractive effect. From the reflectivity changes associated to the Kerr effect we have determined a consistent value for the non-linear refractive index $\mathrm{LiNbO}_{3}$ at $400 \mathrm{~nm}$. Also, the observation of this instantaneous effect provides an accurate determination of the zero delay between pump and probe which enables confirming that the free carriers density reaches its maximum value $550 \mathrm{fs}$ after the pump pulse peak. This unambiguously corroborates that even for transformlimited pulses, $130 \mathrm{fs}$, multiphoton ionization is not the only excitation mechanism, but also delayed impact ionization contributing to free electron generation. We have also observed transient Newton rings in the 100 ps delay range, suggesting that the ablation mechanism is similar to that observed in semiconductors and metals. Finally, our imaging approach has enabled a complete determination of relaxation 
time of the photorefractive effect for a broad range of fluences, from far below up to close to the ablation threshold.

\section{ACKNOWLEDGMENTS}

This work has been partially supported by the Spanish TEC2011-22422 project. M.G.-L. and J.H.-R. acknowledge FPU and FPI grants, respectively, awarded by the Spanish Ministries of Education and of Economy and Competitiveness.

${ }^{1}$ L. Arizmendi, Phys. Status Solidi 201, 253 (2004).

${ }^{2}$ E. G. Gamaly, S. Juodkazis, V. Mizeikis, H. Misawa, A. V. Rode, and W. Krolikowski, Phys. Rev. B 81, 054113 (2010).

${ }^{3}$ S. Sasamoto, J. Hirohashi, and S. Ashihara, J. Appl. Phys. 105, 083102 (2009).

${ }^{4}$ O. Beyer, D. Maxein, T. Woike, and K. Buse, Appl. Phys. B 83, 527 (2006).

${ }^{5}$ O. Beyer, D. Maxein, K. Buse, B. Sturman, H. Hsieh, and D. Psaltis, Phys. Rev. E 71, 056603 (2005).

${ }^{6}$ P. Reckenthaeler, D. Maxein, T. Woike, K. Buse, and B. Sturman, Phys. Rev. B 76, 195117 (2007).

${ }^{7}$ H.-T. Hsieh, D. Psaltis, O. Beyer, D. Maxein, C. von Korff Schmising, K. Buse, and B. Sturman, Opt. Lett. 30, 2233 (2005)

${ }^{8}$ B. Sturman, O. Beyer, D. Maxein, and K. Buse, J. Opt. Soc. Am. B 24, 419 (2007)

${ }^{9}$ Femtosecond Laser Micromach, edited by R. Osellame, G. Cerullo, and R. Ramponi (Springer, Berlin/Heidelberg, 2012), Chapters 1 and 2 and references therein pp. 1-41.

${ }^{10} \mathrm{~A}$. Wu, I. Chowdhury, and X. Xu, Phys. Rev. B 72, 085128 (2005).

${ }^{11}$ V. Temnov, K. Sokolowski-Tinten, P. Zhou, A. El-Khamhawy, and D. von der Linde, Phys. Rev. Lett. 97, 237403 (2006).

${ }^{12}$ B. Rethfeld, Contrib. Plasma Phys. 47, 360 (2007).

${ }^{13}$ D. Puerto, J. Siegel, W. Gawelda, M. Galvan-Sosa, L. Ehrentraut, J. Bonse, and J. Solis, J. Opt. Soc. Am. B 27, 1065 (2010).

${ }^{14}$ S. Guizard, N. Fedorov, A. Mouskeftaras, S. Klimentov, and C. Phipps, in HPLA 2010 (AIP Publishing, 2010), pp. 336-346.
${ }^{15}$ M. Lebugle, N. Sanner, N. Varkentina, M. Sentis, and O. Utéza, J. Appl. Phys. 116, 063105 (2014).

${ }^{16}$ K. Sokolowski-Tinten, J. Bialkowski, A. Cavaleri, D. Von Der Linde, A. Oparin, J. Meyer-ter-Vehn, and S. I. Anisimov, Phys. Rev. Lett. 81, 224 (1998).

${ }^{17}$ B. Rethfeld, V. V. Temnov, K. Sokolowski-Tinten, S. I. Anisimov, and D. Von Der Linde, Proc. SPIE 4760, 72 (2002).

${ }^{18}$ M. Garcia-Lechuga, J. Siegel, J. Hernandez-Rueda, and J. Solis, e-print arXiv:1405.5419 (2014).

${ }^{19}$ M. Garcia-Lechuga, J. Siegel, J. Hernandez-Rueda, and J. Solis, "Femtosecond laser ablation of dielectric materials in the optical breakdown regime: Expansion of a transparent shell," Appl. Phys. Lett. (to be published).

${ }^{20}$ J. Hernandez-Rueda, J. Siegel, M. Galvan-Sosa, A. Ruiz de la Cruz, and J. Solis, J. Opt. Soc. Am. B 30, 1352 (2013).

${ }^{21}$ R. Trebino, K. W. DeLong, D. N. Fittinghoff, J. N. Sweetser, M. A. Krumbügel, B. A. Richman, and D. J. Kane, Rev. Sci. Instrum. 68, 3277 (1997).

${ }^{22}$ J. M. Liu, Opt. Lett. 7, 196 (1982)

${ }^{23}$ D. Puerto, W. Gawelda, J. Siegel, J. Bonse, G. Bachelier, and J. Solis, Appl. Phys. A 92, 803 (2008).

${ }^{24}$ R. L. Fork, O. E. Martinez, and J. P. Gordon, Opt. Lett. 9, 150 (1984).

${ }^{25}$ J. Hernandez-Rueda, D. Puerto, J. Siegel, M. Galvan-Sosa, and J. Solis, Appl. Surf. Sci. 258, 9389 (2012).

${ }^{26}$ D. E. Zelmon, D. L. Small, and D. Jundt, J. Opt. Soc. Am. B 14, 3319 (1997).

${ }^{27}$ H. Li, F. Zhou, X. Zhang, and W. Ji, Appl. Phys. B Lasers Opt. 64, 659 (1997).

${ }^{28}$ C. Quoix, G. Hamoniaux, A. Antonetti, J.-C. Gauthier, J.-P. Geindre, and P. Audebert, J. Quant. Spectrosc. Radiat. Transf. 65, 455 (2000).

${ }^{29}$ K. J. Wædegaard, D. B. Sandkamm, A. Mouskeftaras, S. Guizard, and P. Balling, Europhys. Lett. 105, 47001 (2014).

${ }^{30}$ Y. Qiu, K. B. Ucer, and R. T. Williams, Phys. Status Solidi 2, 232 (2005).

${ }^{31}$ D. Berben, K. Buse, S. Wevering, P. Herth, M. Imlau, and T. Woike, J. Appl. Phys. 87, 1034 (2000).

${ }^{32}$ S. Ashihara, S. Sasamoto, and J. Hirohashi, SPIE Proc. 7197, 719712 (2009). 